\title{
INFLUENCE OF CURVATURE IN REGENERATIVE COOLING SYSTEM OF ROCKET ENGINE
}

\author{
Y. Torres, L. Stefanini, and D. Suslov
}

Thermomechanical loads in rocket engines can be drastically reduced by a reliable cooling system. The regenerative cooling system uses propellants as coolant which flows through milled cooling channels in the chamber walls. Due to centrifugal forces, dynamic secondary motions appear in cooling-channel curvatures, which strongly modify heat transfer. Three-dimensional (3D) numerical calculations have been performed in order to compare this heat flux modification with empirical correlations. Different turbulence models and wall treatments have been tested to develop a complete numerical data base about asymmetrical (concave side) heat transfer in curved cooling channels of rocket engine.

\section{INTRODUCTION}

For an optimal design of rocket-engine cooling systems with minimal hydrodynamic losses, precise knowledge of heat transfer processes in a combustion chamber and in cooling channels is necessary. The combustion chamber imposes some curvature to the cooling channels which are milled in the chamber walls. The maximal heat flux density is reached through the walls of the nozzle throat of rocket engine (up to $80 \mathrm{MW} / \mathrm{m}^{2}$ for the Ariane 5 main-engine radial heat flux). The case of asymmetrical heating at the concave side of cooling channels has been studied here corresponding to the nozzle throat section in a real engine.

Nowadays, 3D numerical simulation could be considered as the most efficient method to determine mechanical, thermal, and pressure loads on a structure, with relatively minimal expense and requirement of time. The main advantage of this computing method is the universal character and the relative precision of the results which increases with the recombination of several sources of information such as existing simulations, experiments and empirical correlations. A Computational Fluid Dynamic (CFD) simulation has to be extended over the entire operating domain to test the robustness of the model: the influence of several parameters has to be characterized and evaluated, like boundary conditions, turbulence models, wall treatment, grid precision, fluid models, wall roughness, etc. 


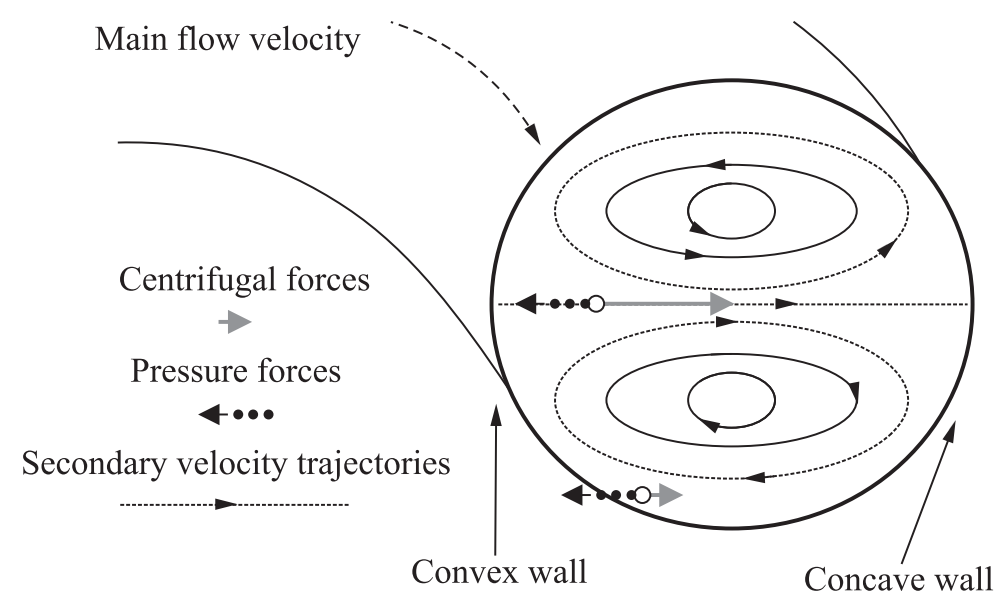

Figure 1 Creation of the Dean vortices in a curved bend

This study focuses on a particular numerical test case that concerns interaction between dynamic secondary flows due to centrifugal acceleration and asymmetrical heat transfer in a high-aspect-ratio cooling channel. An actual experimental realization at DLR delivers the boundary conditions and the geometry of the asymmetrical heated cooling channel for this numerical test case.

The centrifugal acceleration induces a pressure gradient from the concave wall to the convex one. By neglecting the centrifugal forces near the wall region (no main flow velocity region induces no centrifugal forces), the variation of the centrifugal forces to pressure gradient ratio in a channel section implies the formation of counter vortices in the entire cross section of the channel: the Dean vortices (Fig. 1) [1].

These secondary motions can be characterized by a dimensionless number, the Dean number [1]:

$$
\operatorname{De}=\operatorname{Re} \sqrt{\kappa} ; \quad \kappa=\frac{D_{h}}{2 R_{c}} .
$$

Moreover, highly asymmetrical heating imposes a thermal stratification along the channel, clearly demonstrated by experimental and numerical investigations $[2,3]$. The presence of the Dean secondary vortices in heated channel perturbs the thermal stratification, increases mixing and enhances heat transfer at the concave side. The global heat transfer is then locally perturbed and the local Nusselt number at the concave side increases (up to $+25 \%$ ) $[4,5]$.

Some experimental data have been collected concerning the heat transfer through curved cooling channels, and classic empirical correlations have then been elaborated to characterize the enhancement of the heat flux due to the curvature. Experimental investigations have been provided for rocket engine $[6$, 
7], and Niino and Kumakawa have adapted the initial empirical correlation of Taylor [8]:

$$
\begin{aligned}
& \frac{\mathrm{Nu}_{\text {curved }}}{\mathrm{Nu}_{\text {straight }}}=\left[\mathrm{Re} \cdot \kappa^{2}\right]^{ \pm 0.05}[8] ; \\
& \frac{\mathrm{Nu}_{\text {curved }}}{\mathrm{Nu}_{\text {straight }}}=\left[\mathrm{Re} \cdot \kappa^{2}\right]^{ \pm 0.02}\left[1+\frac{1}{3} \sin \left(\pi \sqrt{\frac{x_{c}}{L_{c}+15 D_{h}}}\right)\right][6] ; \\
& \frac{\mathrm{Nu}_{\text {curved }}}{\mathrm{Nu} \text { straight }}=\left[\mathrm{Re} \cdot \kappa^{2}\right]^{ \pm 0.05 \sin \left(\pi \sqrt{x_{c} /\left(L_{c}+37 D_{h}\right)}\right)}
\end{aligned}
$$

The sign "+" of the preceding correlations is used to determine the heat transfer enhancement at the concave side of the curved cooling channel, although the sign "-" is for the decrease of the heat transfer at the convex side.

Nevertheless, it can be noted that the extreme thermal, fluid, and mechanical real conditions (high heat flux density, cryogenic propellants, complex mechanical construction, etc.) bring some difficulties in obtaining similar and reliable experimental results. Some experimental realizations have been set up on single cooling channel, electrically heated, advantaging sensors implementation, accessible experimental conditions, and reliability [4, 9]. However, the domain of experimental realization is far away from the real rocket-engine conditions (microdimension of channels, high heat transfer, cryogenic hydrogen, high aspect ratio of micro-channel).

The following correlation comes from experimental investigation [5] on an electrical heated channel:

$$
\frac{\mathrm{Nu}_{\text {curved }}}{\mathrm{Nu}}=\mathrm{Re}_{\text {straight }} \mathrm{e}^{0.046} \kappa^{0.1} \text {. }
$$

Numerical simulations have been performed, with different methods of calculation and for different test configuration (real cooling channels of rocket engine [3, 10], straight channels [11], referent test case [12]). These numerical references are part of a project at DLR Lampoldshausen: the EH3C project (Electrical Heated Curved Cooling Channels). Concerning the numerical work of this project (presented in this paper), an interesting comparison can be done with the preceding empirical correlations. All these numerical simulations have taken the experimental conditions of a campaign that will be published later. Numerical simulations of the EH3C project have been carried out using 3D CFD calculation, performed with CFX 10.0.

Several parameters have been investigated including grid convergence, fluid and solid model, roughness, some boundary condition parameters and finally turbulence models and near-wall treatments. 


\section{NUMERICAL SIMULATIONS}

To characterize the turbulent variations, the thermal and dynamical parameters are decomposed into a time averaged component and temporal fluctuation component. Then, the basic equations have been time averaged: the ReynoldsAveraged Navier-Stokes (RANS) equations have been used in this research to model the turbulence. Some closure equations are needed to solve the equations system. The choice of the right turbulence model and the right wall treatment represents one of the most important challenges for engineering numerical applications, to save time costs and to allow representations of dynamics and heat fluxes close to reality. Turbulence has been here modeled with two-equation $(k-\varepsilon, k-\omega)$ closure systems and seven-equation models (Reynolds Stress Model, RSM). The 2-equation closure systems of turbulence use the Boussinesq assumption to calculate the Reynolds stress tensor which is a linear, isotropic, and homogeneous model. The turbulent component (Eddy viscosity concept) of the viscosity is then directly deduced from the 2-equation closure model.

A large number of scientific and engineering calculations adhering to turbulent flows are established on the $k-\varepsilon$ model, complemented by a logarithmic function to patch the core region of the flow in the wall region. The $k-\omega$ model [13] seems to be well adapted to conjugate heat and mass transfer. Nevertheless, the $k-\omega$ model would present strong sensitivity to free-stream conditions that can be improved by the good compromise of a Shear Stress Transport (SST) model. This SST turbulence model uses a blending function to apply the $k-\varepsilon$ model in the bulk region and the $k-\omega$ inside the boundary layer [14].

Other kinds of closure models do not adopt the Boussinesq assumption and allow a nonlinear resolution of the Reynolds stress tensor that gives an anisotropic model. This is quite well adapted to approach recirculation, high shear-stress gradient regions, and curvatures but needs an important convergent time (7equation resolution instead of 2) and the convergence of calculations is not so stable as for the 2-equation models. Except from the resolution of the Reynolds stresses, the particular BSL RSM ("Baseline Reynolds Stress Model") has been used here and is based on an $\omega$-equation but integrating blending coefficients from the $k$-equation.

There are two different approaches for the near-wall treatment. First, there are "wall functions," in which the viscosity-affected inner region is not numerically calculated but replaced by semiempirical formulas to bridge the viscosityaffected region between the wall and the fully-turbulent region. The other approach is called Low-Reynolds Model (LRM), in which the turbulence models are modified to enable the viscosity-affected region to be resolved with a precise mesh near the wall, including the dynamic resolution of the viscous sublayer (where Reynolds number is low). This method is more precise than the logarithmic approximation but requires much more cells near the wall domains and needs more calculation time. 


\section{NUMERICAL MODEL}

The curvature effects on asymmetrical heat transfer in rocket cooling channels are investigated numerically and experimentally at the DLR of Lampoldshausen with the project EH3C. The experimental setup concerns manufactured test specimens: one single straight channel and one curved concave channel to study the influence of Dean vortices on heat transfer. The experimental conditions are close to the real-engine channels conditions: the coolant is supercritical cold hydrogen: 40 bar and $80 \mathrm{~K}$ at the entrance of the cooling channel. The asymmetrical heat flux is about $20 \mathrm{MW} / \mathrm{m}^{2}$, created from electrical heaters delivering up to $20 \mathrm{~kW}$. Moreover, the EH3C channels reflect real geometry of real engine channels [2] (high aspect-ratio channels). The obtained Reynolds number in this test case reaches $3 \cdot 10^{5}\left(\sim 2 \cdot 10^{6}\right.$ for Vulcain configuration) and the Dean number is about $2 \cdot 10^{4}$.
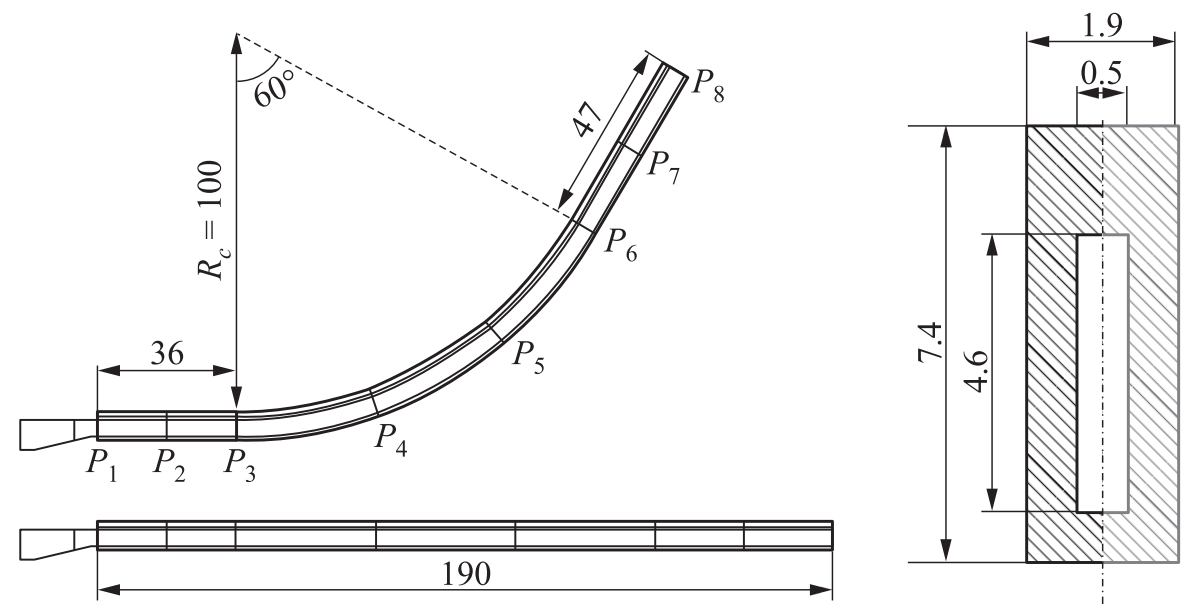

Figure 2 Geometry of the EH3C test specimen. Dimensions are in millimeters

Figure 2 describes the geometry of the 2 test specimens which have been numerically simulated.

The numerical geometry only models the half of the channel for reasons of symmetry. The entrance of the channel has been modeled as well: a convergence at the inlet allows a smooth growth of the thermal and dynamic boundary layers. Several plane surfaces (P1 to P8) are located along the channel to illustrate the results.

The boundary conditions used for the numerical model follow the experimental conditions of the EH3C campaign as it is shown in Fig. 3: 
Heat flux density along channel perimeter

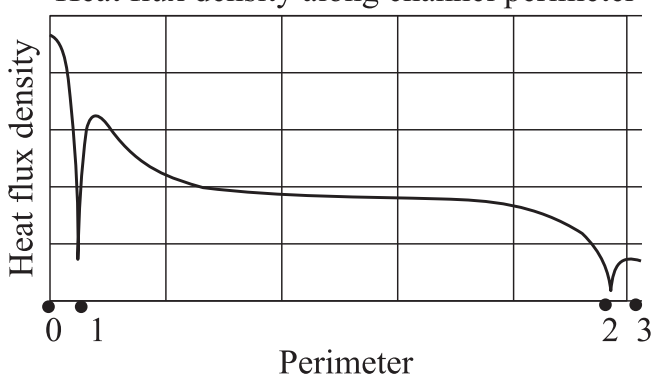

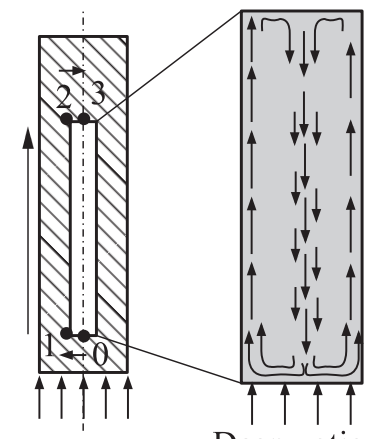

Dean votices orientation
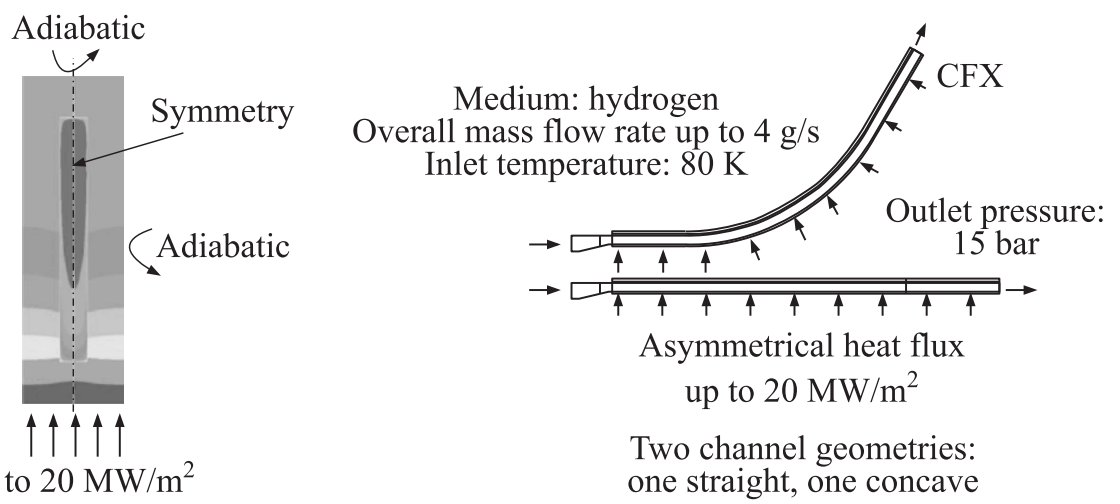

Two channel geometries: one straight, one concave

Figure 3 Boundary conditions of the numerical model and typical heat transfer profile along channel perimeter

- mass flow rate $4 \mathrm{~g} / \mathrm{s}$ at the inlet $(2 \mathrm{~g} / \mathrm{s}$ for the modeled half of channel);

- pressure 15 bar at the outlet;

- symmetry on the median lines;

- heat flux density $20 \mathrm{MW} / \mathrm{m}^{2}$ on the lower wall;

- adiabatic condition on the other external walls;

- hydrogen as fluid;

- copper alloy as solid; 
- inlet premixing turbulence at $1 \%$ with an Eddy Length Scale of $0.5 \mathrm{~mm}$; and

- the roughness has been measured on the manufactured channels and implemented in the numerical model.

A typical way to analyse results from the numerical simulation is presented in Fig. 3. The profile of the heat flux density is drawn around the channel walls. Indeed, for any comparisons, the profile of heat flux density along channel periphery seems to be the most appropriated parameter to compare straight and curved simulations (see Fig. 3).

The inlet turbulence conditions have been changed (from $1 \%$ to $10 \%$ with varying Eddy length scales) and do not have any influence on the numerical results. The averaged roughness is included in the viscous sublayer and does not have any influence on the thermal and dynamic behavior. This has been proven by different numerical simulations with and without roughness.

Several grids with different number of nodes have been designed in order to check the grid convergence. All the calculations used to make this comparison were carried out using a standard $k-\varepsilon$ model with a wall function near-wall treatment. The entire grid is structured, both in solid and fluid regions. To ensure better fluid and thermal resolutions in high gradient domains (near to walls fluid domains), conformal mesh interfaces have been adopted for the fluid/solid and the fluid/fluid interfaces in the channel. All the solid/solid interfaces are nonconformal to reduce the number of nodes in the solid outer region while keeping the global accuracy of the simulations (Fig. 4).

Two kinds of grids have been designed either for using a wall function (first fluid cell in the fully turbulent region of the boundary layer) or for using an LRM (around 5 cells inside the viscous sublayer). The solid part (channel wall) has been divided into two zones: an inner part in contact with the fluid insuring conform interfaces and an outer part all around. In the inner solid zone, a matching number of nodes was set and the outer solid region used a coarser grid. The first grid, with globally 1 million of nodes, is a $60 \times 15$ grid with 530 nodes streamwise in the fluid zone (Mesh 1).

Some other grids present alternate distribution of the nodes streamwise (Mesh 2) with a finer distribution inside the curvature, or global refinement in the cross section (Mesh 3). Mesh 4 increases the streamwise number of nodes. This has been done to check the influence of the cell aspect ratio (streamwise length on height of cell) and of the mesh refinement on the results. The change of the streamwise cells distribution does not have a strong influence on the results, and the streamwise number of nodes has been limited to 530 with a homogeneous repartition along the cooling channel. Another grid has been developed $120 \times 20 \times 530$ grid, with a total of 2.5 million nodes and has been designed to observe the influence of the cross section meshing on results (Mesh 5). The heat flux profiles from the different grids seem to converge with nodes increasing in 

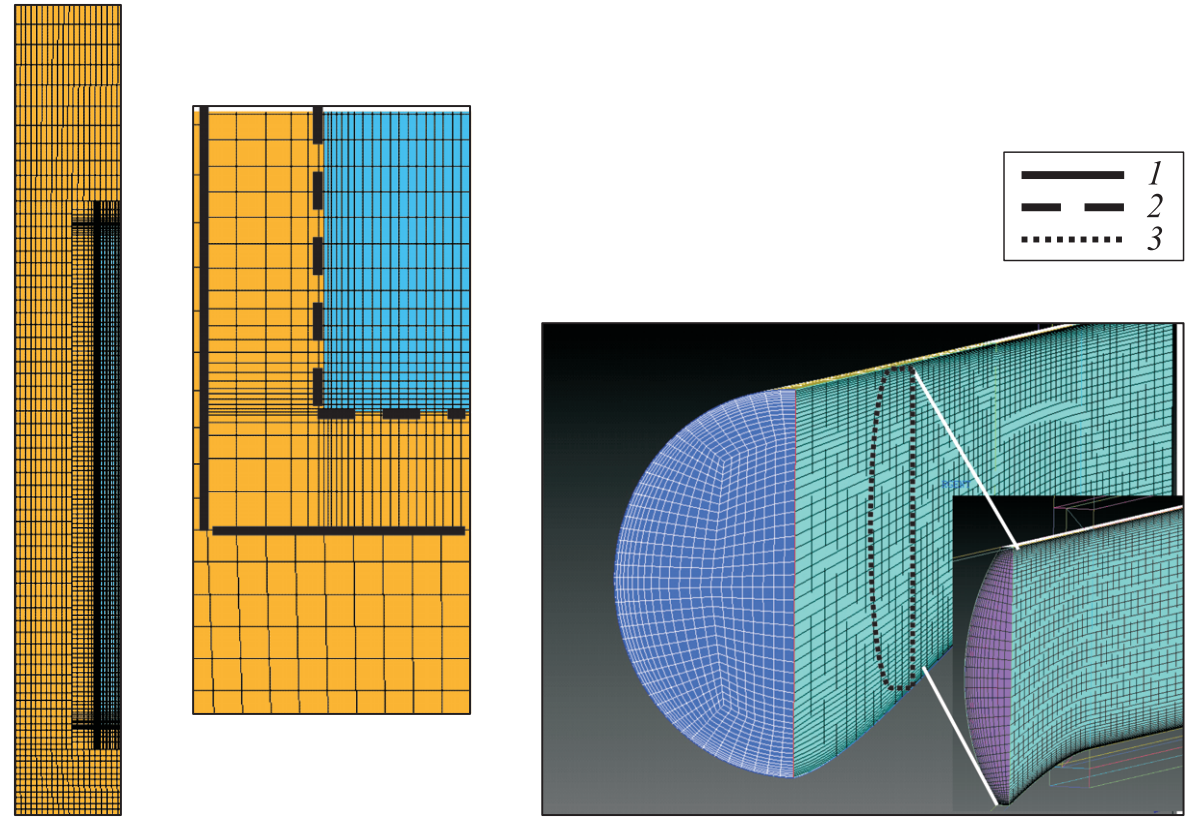

Figure 4 Meshing of the single cooling channel (Mesh 5): 1 - conform fluid/solid interfaces; 2 - nonconform fluid/solid interfaces; and 3 - conform fluid/fluid interfaces. (Refer Torresetal., p. 178.)

the cross section, particularly after the curvature. Then, the finest mesh looks appropriate for this numerical model (Mesh 5) and has been selected for all further numerical investigations. The difference of the heat flux profile does not vary more than $5 \%$ between Meshes 4 and 5 . The LRM requires a finer grid

Table 1 Description of the different meshes

\begin{tabular}{lrccccc}
\hline $\begin{array}{c}\text { Cross } \\
\text { section }\end{array}$ & Nodes & $\begin{array}{c}\text { Streamwise, } \\
\text { nodes }\end{array}$ & $\begin{array}{c}\text { Maximum } \\
\text { cell } \\
\text { aspect } \\
\text { ratio }\end{array}$ & $\begin{array}{c}\text { Nodes, } \\
\text { mln }\end{array}$ & $\begin{array}{c}\text { CPU time, } \\
\text { h }\end{array}$ & $y^{+}$ \\
\hline Mesh 1 & $60 \times 15$ & 530 & 64 & 1 & 5 & $12-70$ \\
Mesh 2 & $60 \times 15$ & 530 & 50 & 1 & 5 & $12-70$ \\
Mesh 3 & $80 \times 20$ & 530 & 60 & 1.7 & 7 & $12-70$ \\
Mesh 4 & $80 \times 20$ & 1170 & 23 & 3.6 & 17 & $12-70$ \\
Mesh 5 & $120 \times 20$ & 530 & 60 & 2.5 & 12 & $12-70$ \\
LRM & $140 \times 40$ & 530 & 770 & 4.5 & 60 & $0.15-2.5$ \\
\hline
\end{tabular}


near the walls, so an additional grid has been designed, to have $y^{+}$between 0.5 and 2 on the entire fluid/solid interface, reaching a grid of 4.5 millions nodes. The grid convergence study on the meshing of the LRM grid is unfortunately limited by calculation time requirements. The details of all investigated grids are presented in Table 1. The CPU time has been evaluated in hours on a parallel nodes cluster.

\section{EFFECTS OF CURVATURE}

The effects of curvature on the heat transfer in a cooling channel are significant. The straight and the curved models have been designed exactly with the same grid and the same boundary conditions. The first comparison between the straight and curved cases were carried out with the ideal gas model (hydrogen), constant properties of the solid (copper alloy), and a $k-\varepsilon$ turbulence model.

In plane 3 (P3 referred in Fig. 2), just before the curvature of the curved channel, straight and curved cases provide the same heat flux distribution with a difference of less than $2 \%$. The two test cases can be then compared and the differences are then only due to the centrifugal forces. Supplementary pressure losses are added for the curved case, due to the energy dissipation of the secondary motions in the curved channel. However, the pressure losses for the straight channel reach 19.5 bar and for the curved case 19.9 bar, that is not so important.

The curvature induces Dean vortices: the 'cold' fluid coming from the center of the channel is displaced to the "hot" concave wall (Fig. $5 a$, right). Then, the fluid coming from the "hot" wall is moving up along the lateral walls, always taking into account the no-slip condition at the wall.

In the cross section 5 (P5) which is located in the curvature, the heat flux distribution is radically different. A much higher heat flux density is observed on the lower wall (Fig. 5b). Indeed, the heat flux density is enhanced through the concave wall (the heated one in this case) and reduced through the convex wall. This explains the decreasing of the local heat flux through the lateral walls near the concave side because of hotter fluid (already heated by the concave side).

In these simulations, the global heat flux is constant for any axial sections along both channels. Then, both cooling channels absorb the same heat $7.22 \mathrm{~kW}$. Only the distribution of the heat flux density changes around the perimeter of the channel by comparing the curved to straight configurations. The next calculations were carried out with a real gas fluid model (Redlich-Kwong model) and an adequate model of the copper alloy has been used (thermal conductivity). Several turbulence models have been tested in straight and curved channels. Both Near-Wall Treatment models have been investigated, using either SST or 


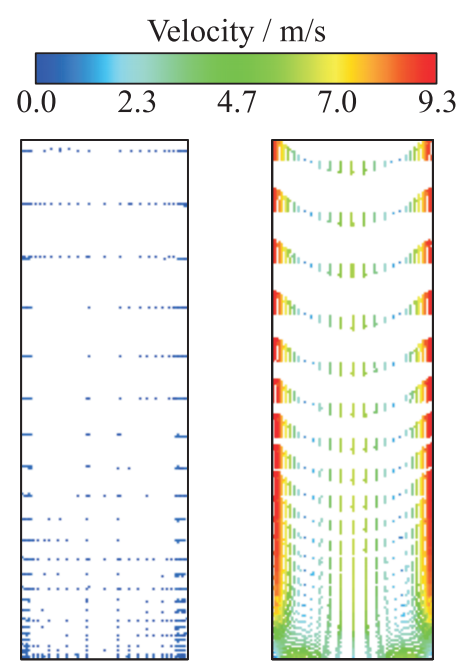

(a)

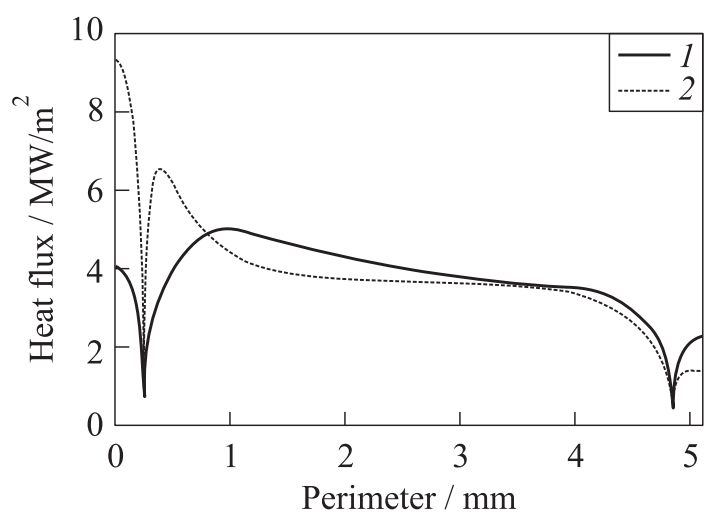

(b)

Figure 5 (a) Secondary velocities calculated in a straight channel (left) and in a concave channel (right) (colored by velocity magnitude); and (b) heat flux distribution for straight (1) and curved (2) cases in plane 5. (Refer Torres et al., p. 180.)

RSM turbulence model. The results of the numerical simulation are presented in Fig. 6.

All the turbulence models detect the presence of the Dean vortices but indicate several intensities and several shapes of these secondary motions. Moreover, with the RSM model, additional secondary motions are visible near the corners of the straight (Fig. $7 a$, left) and curved (Fig. $7 a$, right) channels which interact with the Dean vortices in the curved-channel simulation. This does not appear for all other 2-equation models. In fact, in rectangular channels, the orthogonal walls bring shear stress asymmetries. These vortices can be compared to those observed in Large Eddy Simulation (LES) [11] (Fig. 7b) and are in fact very similar in shape and intensity, despite a RANS model is used there. The LES have been provided with a model of isothermal channel and asymmetrical heated cooling channel. Both models let appear the corner vortices.

By taking into account the anisotropy of the turbulence, the RSM turbulence models seem to be the most adapted models for the numerical calculations of rectangular channels. In the curvature, the mixing of the Dean vortices and the "corner vortices" are shown in Fig. $7 a$, right.

The Nusselt numbers ratio (curved to straight) has been calculated on the concave side (center of the channel) by different ways: empirical correlations 


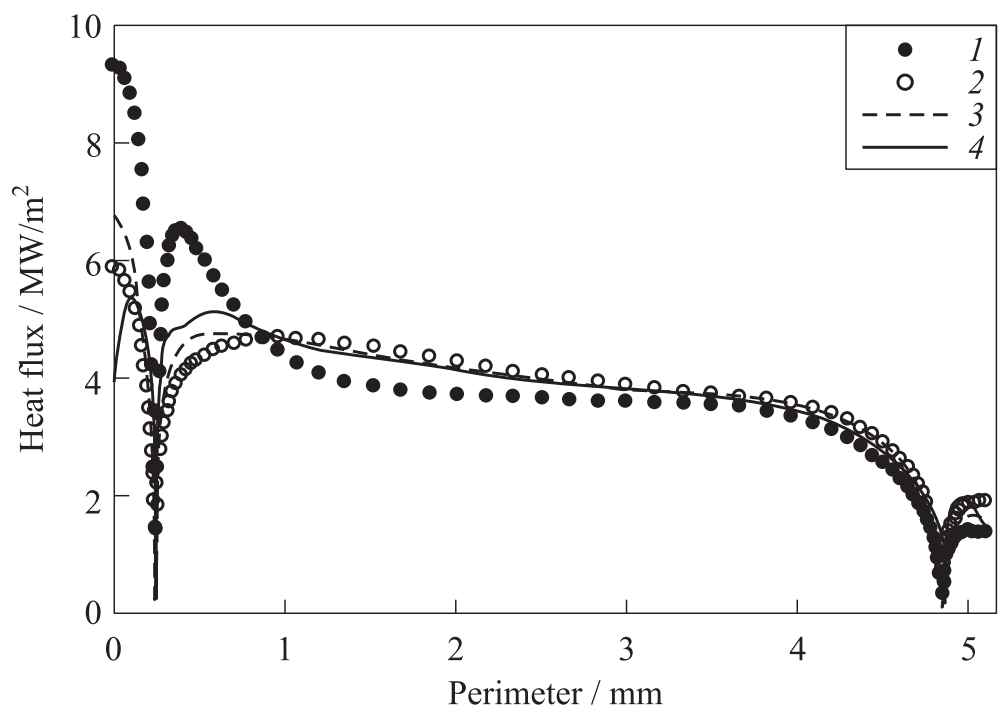

Figure 6 Heat flux distribution along the perimeter for different wall treatments and turbulence models at the section P5 of the curved channel: $1-k-\varepsilon ; 2-\mathrm{SST} ; 3-$ SST LRM, and $4-$ RSM LRM

and numerical simulations (Fig. 8). The curvature begins at $36 \mathrm{~mm}$ after the entrance and ends at $141 \mathrm{~mm}$ along the channel.

With the EH3C test case conditions, the available empirical correlations are varying between $8 \%$ and $40 \%$ local heat transfer increase at the concave wall. The numerical simulations indicate an increase of $15 \%$ up to $40 \%$.

The main deficiency of empirical correlation is the limited range where they are available and their adaptation to different experimental domain (pressure range, fluid, dimensions, heat flux range, temperature ranges, etc.).

Concerning the numerical simulations, the variation due to turbulence models can reach locally $50 \%$ and this uncertainty comes principally from the choice of the turbulence model.

The RSM calculation exhibits "in stair" behavior along the channel. This is explained by the interaction of the "edge vortices" with Dean vortices.

Indeed, it has been noted that the Dean vortices and the upper half vortex from the edge are not distinct anymore (Fig. $7 a$, right) and the "edge vortices" seem to disappear along the curvature (here, at the abscissa 80 in Fig. 8), giving a full access of the Dean cells to the concave side, creating a "delayed" heat transfer increase along the channel axis. 


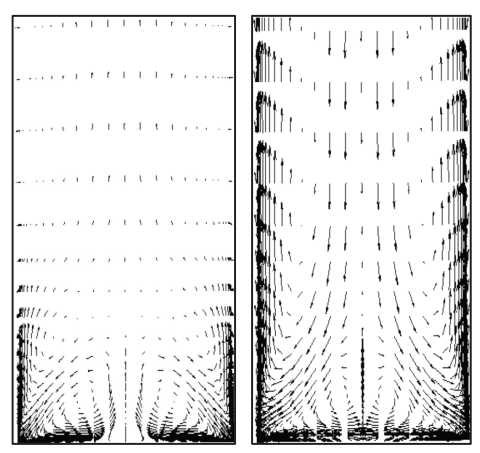

(a)
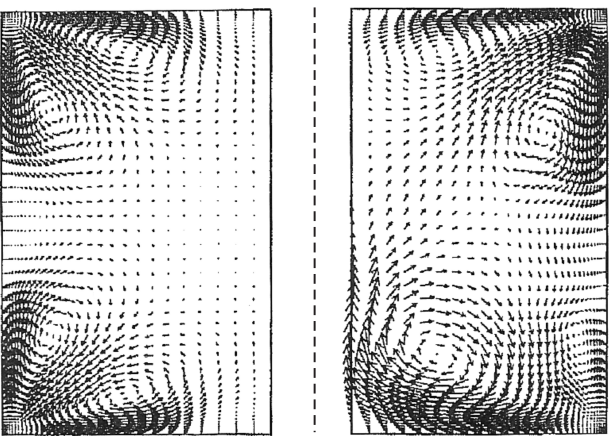

(b)

Figure 7 (a) Secondary velocity distribution in plane 5 in straight (left) and curved (right) channel calculated with an RSM model from these EH3C simulations; and (b) LES of turbulent flow in a square straight channel [11] with isothermal condition (bulk-to-wall temperature ratio $T_{b} / T_{w}=1$, left) and heated lower side condition $\left(T_{b} / T_{w}=2.5\right.$, right $)$

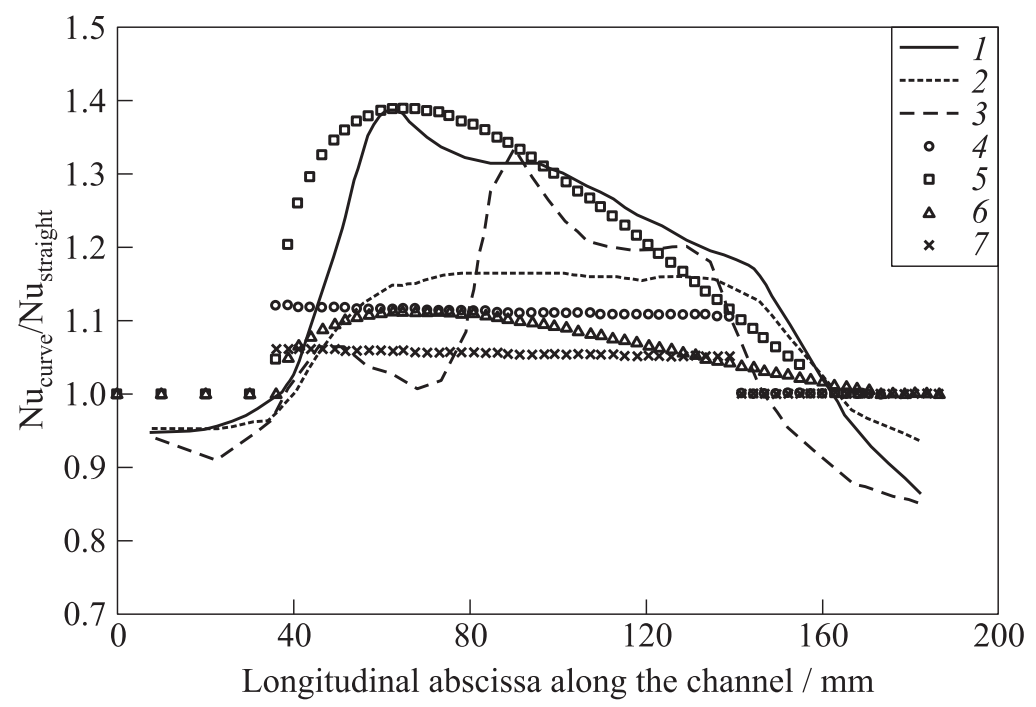

Figure 8 Curved-to-straight Nusselt ratio along channel length: $1-k-\varepsilon ; 2-$ SST; $3-\mathrm{RSM} ; 4-[8] ; 5-[6] ; 6-[7]$; and $7-[5]$ 


\section{CONCLUDING REMARKS}

Numerical simulations have pointed out the important role of the turbulence anisotropy in cooling-channel flow calculation. The RSM seems to be the most appropriate model for taking into account "edge recirculation." Several RSM options have now to be tested and improved to check the robustness of these nonisotropic models ( $\varepsilon$ or $\omega$ basic equation, BaseLine, Isotropization of Production model, Quasi-Isotropic model, etc.). The role of heat transfer is an interesting parameter to be investigated, the Dean-"edge" vortex interaction could depend on the intensity of thermal stratification at the concave side of the cooling channel.

\section{REFERENCES}

1. Dean, W. R. 1927. Note on the motion of fluid in a curved pipe. M.A., Imperial College of Science, Philos. Mag. IV: 208.

2. Woschnak, A., D. Suslov, and M. Oschwald. 2003. Experimental and numerical investigations of thermal stratification effects. AIAA Paper No. 2003-4615.

3. Wennerberg, J. C., J. Hogirl, R. Schuff, W. E. Anderson, and C. L. Merkle. 2006. Study of simulated fuel flows in high aspect ratio cooling channels. AIAA Paper No. 2006-4708.

4. Neuner, F., D. Preclik, M. Popp, M. Funke, and H. Kluttig. 1998. Experimental and analytical investigation of local heat transfer in high aspect ratio cooling channels. AIAA Paper No. 98-3439.

5. Sturgis, J. C., and I. Mudawar. 1999. Single-phase heat transfer enhancement in a curved, rectangular channel subjected to concave heating. Int. J. Heat Mass Transfer 42(7):1255-72.

6. Niino, M., A. Kumakawa, N. Yatsuyanagi, and A. Suzuki. 1982. Heat transfer characteristics of liquid hydrogen as a coolant for $\mathrm{LO}_{2} / \mathrm{LH}_{2}$ rocket thrust chamber with the channel wall construction. National Aerospace Lab of Japan. Ohgawara, Miyagi, Japan. AIAA/SAE/ASME, 18th Joint Propulsion Conference. Cleverland, Ohio.

7. Kumakawa, A., M. Saski, M. Niino, H. Sakamoto, and T. Sekita. 1986. Thermal conduction characteristics of an electrically heated tube modeld after the LE-7 main burner. 30th Space Sciences and Technology Conference.

8. Taylor, M. F. 1968. A method of predicting heat transfer coefficients in the cooling passages of Nerva and Phoebus-2 rocket nozzle. AIAA Paper No. 68-608.

9. Meyer, M. 1995. Electrically heated tube investigation of cooling channel geometry effects. AIAA Paper No. 95-2500.

10. Fröhlich, A., H. Immich, F. LeBail, and M. Popp. 1991. Three-dimensional flow analysis in a rocket engine coolant channel of high depth/width ratio. AIAA Paper No. 91-2183. 
11. Salinas Vasquez, M., and O. Métais. 2002. Large-eddy simulation of the turbulent flow through a heated square duct. J. Fluid Mech. 453:201-38.

12. Münch, C., and O. Métais. 2005. Turbulence in cooling channels of rocket engines, Large Eddies Simulation. Comptes Rendu de Mécanique de l'Académie des Sciences 333:574-79.

13. Wilcox, D. C. 1986. Multiscale model for turbulent flows. AIAA 24th Aerospace Sciences Meeting. American Institute of Aeronautics and Astronautics.

14. Menter, F. R. 1994. Two-equation eddy-viscosity turbulence models for engineering applications. AIAA J. 32(8):1598-1605. 